\title{
ESTIMAÇÃO INDIRETA DE VELOCIDADE DE UM MOTOR DE INDUÇÃO TRIFÁSICO UTILIZANDO UM ANALISADOR DE ESPECTRO LOCK-IN
}

\author{
Eduardo T. F. Santos \\ eduardot@ufba.br \\ Depto. de Engenharia Elétrica, \\ Universidade Federal da Bahia \\ Rua Aristides Novis, 02, Escola \\ Politécnica, Federação \\ CEP : 40210-630, Salvador, BA, \\ BRASIL, +55-71-237-2367
}

\author{
Amauri Oliveira \\ amauri@ufba.br \\ Depto. de Engenharia Elétrica, \\ Universidade Federal da Bahia \\ Rua Aristides Novis, 02, Escola \\ Politécnica, Federação \\ CEP : 40210-630, Salvador, \\ BA, BRASIL, +55-71-237-2367
}

\begin{abstract}
This article discusses the development and utilization of a Digital Lock-in Spectrum Analyzer to estimate the speed of a three-phase induction motor in a less invasive manner. The estimation of speed is obtained using acquisition and processing of one phase-current, without direct physical intervention on the motor. The Digital Lock-in Spectrum Analyzer uses Lock-in Amplifier principle at equally spaced frequencies. The Lock-in Amplifier implements phase-sensitive detection applied to measurement of signals under severe noise conditions.
\end{abstract}

KEYWORDS: induction motor; data acquisition; digital signal processors; Fourier transforms; power spectral density; frequency signal analysis; computational methods; harmonic analysis; speed measurements; tachometers.

\section{RESUMO}

Este artigo trata do desenvolvimento e utilização de um

Artigo Submetido em 16/03/03

1a. Revisão em 06/10/04;

2a. Revisão em 13/09/04

Aceito sob recomendação do Ed.Assoc.Prof.José R. C.Piqueira
Analisador de Espectro Lock-in Digital para estimar a velocidade de um motor de indução trifásico, de maneira não-invasiva, por meio de aquisição e processamento de uma das correntes de fase, sem a necessidade de intervenção física direta no motor. Um Analisador de Espectro Lock-in Digital utiliza o princípio do Amplificador Lock-in para freqüências espaçadas uniformemente, o qual implementa detecção sensível à fase, usada para medição de sinal de freqüência específica em condições adversas de ruído.

PALAVRAS-CHAVE: motor de indução; aquisição de dados; Processador de Sinais Digitais; Transformada de Fourier; Densidade Espectral de Potência; análise de freqüência de sinal; métodos computacionais; análise de harmônicos; Medições de velocidade; tacômetros.

\section{INTRODUÇÃO}

A medição de sinais contaminados com ruído de amplitude maior do que o componente que se deseja medir é um problema muito comum entre pesquisadores experimentais. Técnicas de condicionamento de sinal têm aplicabilidade muito limitada nestes casos. Quando o ruído tem amplitude maior do que o sinal, a abordagem de recuperação de sinal (signal recovery) (Meade, 1983), é mais adequada. 
A recuperação de sinal pode ser realizada através da técnica de detecção sensível à fase (phase-sensitive detection), que permite medir a amplitude de um sinal com freqüência específica.

O dispositivo que realiza a recuperação de sinal por meio da técnica de detecção sensível à fase é denominado Amplificador Lock-in. A limitação do dispositivo convencional de medir um sinal de freqüência específica pode ser eliminada através da varredura, discreta e uniforme, de uma faixa de freqüência, utilizando o dispositivo para medir o sinal em cada uma destas freqüências. Ou seja, pode-se usar o dispositivo modificado como um analisador de espectro de alta resolução que forneça a amplitude de cada freqüência que componha o sinal analisado.

A versatilidade e a flexibilidade dos circuitos digitais, conduzem à conclusão de que a implementação de um Amplificador Lock-in Digital é mais flexível devido ao fato de ser mais fácil alterar o software interno do que modificar os circuitos de um Amplificador Lock-in Analógico. Esta característica da implementação digital foi utilizada para a implementação de um Analisador de Espectro Lock-in a partir de um Amplificador Lock-in Digital.

A partir do espectro de freqüências de uma das correntes de fase do motor de indução trifásico, pode ser estimada indiretamente a velocidade de seu eixo de forma nãoinvasiva (Hurst, 1996 e Blasco-Gimenez, 1996). Realizar a medição de velocidade de forma não-invasiva em regime estacionário, que é a proposta deste artigo, é de grande relevância para motores situados em locais de difícil acesso ou modificação, além de ser mais prática para os demais motores.

O espectro de freqüências é obtido com um dos métodos tradicionais tais como a Transformada de Fourier ou a Densidade Espectral de Potência, como é citado na literatura (Press, 1992). Infelizmente, estes métodos possuem restrições em sua implementação digital, tais como a necessidade de um número de amostras relativamente alto para se obter uma resolução espectral razoável, e a alta sensibilidade a ruídos. Estas restrições limitam a exatidão destes métodos para a medição indireta da velocidade de um motor de indução trifásico.

Através de um Analisador de Espectro Lock-in, torna-se possível obter o espectro de freqüências de uma das correntes de fase com menos amostras, maior resolução espectral e maior imunidade a ruídos do que um dos métodos tradicionais citados. A partir do processamento do espectro de alta resolução obtido é possível estimar, de modo não-invasivo, a velocidade do eixo de um motor de indução trifásico.

\section{AMPLIFICADOR LOCK-IN DIGITAL}

O diagrama de blocos (Meade, 1983) de um Amplificador Lock-in Digital é mostrado na Figura 1 (parte tracejada). O sinal analógico do conversor D/A é injetado no sistema observado, e o sinal de saída deste é obtido por um conversor A/D e processada pelo Amplificador Lock-in Digital para determinação de amplitude, $V$, e de fase, $\phi$.

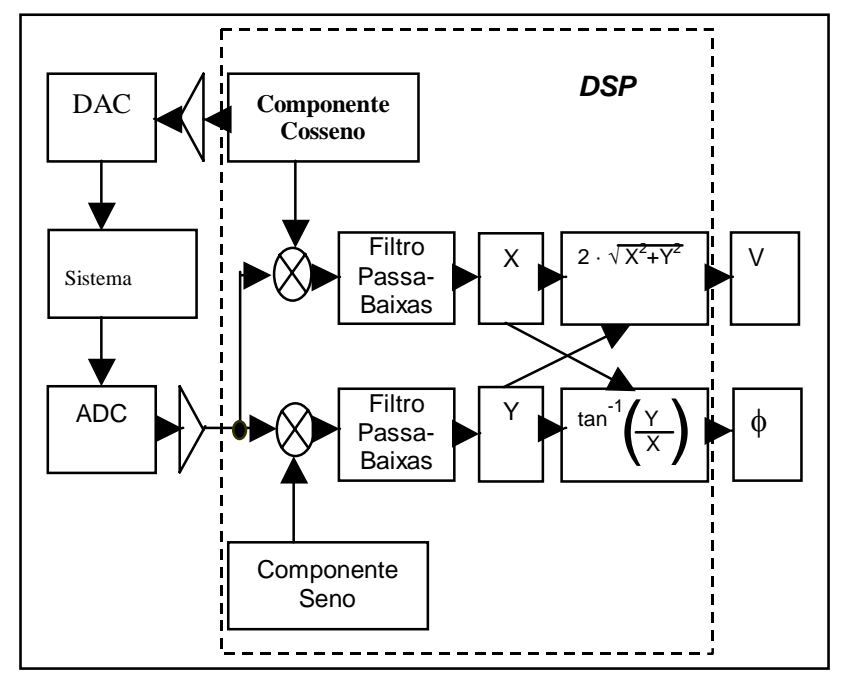

Figura 1 : Diagrama de blocos do Amplificador Lock-in Digital (ALID).

O Amplificador Lock-in Digital realiza as operações de seu equivalente analógico através de software. No lugar de utilizar filtros e operadores aritméticos analógicos, são utilizados filtros digitais e operações aritméticas em software. Os componentes cosseno e seno, respectivamente em fase e quadratura, também são gerados através de software.

A flexibilidade e versatilidade da implementação digital permitem modificar facilmente o modo de operação do Amplificador Lock-in Digital através de alterações no seu software, característica esta empregada para tranformá-lo em um Analisador de Espectro Lock-in.

O sinal recebido do experimento está contaminado com ruído que é assumido como ruído branco (Stark, 1998). O sinal de entrada é multiplicado pelos sinais de referência em fase e quadratura, obtendo-se dois componentes, respectivamente $X$ e $Y$, como pode ser visto na Figura 1 . Esta multiplicação produz uma modulação no sinal, resultando em um componente contínuo (DC) que possui informação sobre o sinal e um ou mais componentes alternados que podem ser desprezados. A eliminação destes componentes alternados permite minimizar ruídos na medição do experimento externo. 
Os sinais $X$ e $Y$ são processados por dois filtros digitais passa-baixa (Tompkins, 1993), os quais permitem que apenas o componente contínuo $(0 \mathrm{~Hz})$ e pequenas variações deste permaneçam, eliminando os componentes alternados. Se as larguras de banda dos filtros são pequenas, a potência do ruído filtrado torna-se desprezível. Nestas condições, os componentes nas saídas dos filtros resultam em (Meade, 1983) :

$$
\begin{aligned}
& X=\frac{V}{2} \cdot \cos (\theta) \\
& Y=\frac{V}{2} \cdot \operatorname{sen}(\theta)
\end{aligned}
$$

Onde $V$ é o valor r.m.s. da saída do sistema externo medido e $\theta$ é a defasagem produzida pelo mesmo. Com estes dois componentes é possível obter os valores da amplitude $V$ e fase $\theta$ através das expressões (Meade, 1983) :

$$
\begin{aligned}
& V=2 \cdot\left(X^{2}+Y^{2}\right)^{1 / 2} \\
& \theta=\tan ^{-1}(Y / X)
\end{aligned}
$$

\section{ANALISADOR DE ESPECTRO LOCK- IN}

O Amplificador Lock-in Digital recupera o sinal de um sistema externo em uma freqüência específica denominada freqüência de referência. Para que o sistema externo forneça uma resposta nesta freqüência, um sinal de referência é gerado para excitar ou modular a entrada do experimento nesta mesma freqüência.

Para implementar um Analisador de Espectro Lock-in Digital, se faz necessário efetuar uma varredura da freqüência do sinal de referência. Ou seja, o Analisador de Espectro Lock-in Digital realiza a recuperação de sinal em uma faixa de freqüências enquanto que o Amplificador Lock-in realiza a recuperação de sinal em uma freqüência única. Devido ao tempo relativamente alto de estabilização dos filtros passa-baixa do Amplificador Lock-in para uma única freqüência, esta operação geralmente não é realizada em tempo real (Meade, 1983).

Neste artigo foi adotada uma modificação da arquitetura do Analisador de Espectro Lock-in apresentada em (Meade, 1983). Ao invés de efetuar a varredura de freqüência ao mesmo tempo em que o processamento do sinal de entrada é realizado (processamento on-line), é efetuada a aquisição de um determinado número $N$ de amostras e só então é efetuada a varredura de freqüências (processamento batch), conforme é mostrado na Figura 2. Este processo é repetido seqüencialmente, alternando entre aquisição e varredura, o que traz como vantagens um espectro consistente sobre um mesmo período de aquisição e um melhor tempo de resposta a alterações rápidas do sinal.

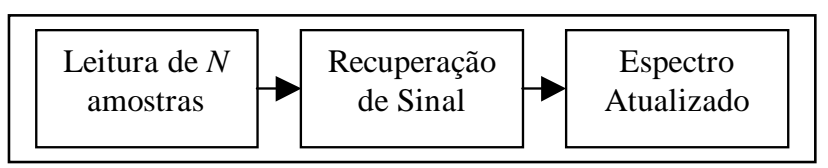

Figura 2 : Fluxograma simplificado do algoritmo do Analisador de Espectro Lock-in Digital

Para um mesmo número de amostras e taxa de amostragem, o Analisador de Espectro Lock-in tem uma resolução espectral maior do que as decorrentes de técnicas convencionais tais como Transformada Rápida de Fourier (FFT) e Densidade Espectral de Potência (PSD). Por exemplo, para uma taxa de amostragem de 8192 Hz e 2048 amostras, a FFT e a PSD possuem resolução espectral de 8192 / 2048 = 4 Hz / divisão (Oppenheim, 1997). Ou seja, o espectro tem medições dos valores das freqüências de 4 $\mathrm{Hz}$ em $4 \mathrm{~Hz}$. Por meio de um Analisador de Espectro Lock-in é possível obter resoluções de $1 \mathrm{~Hz}$. Esta resolução pode ser aumentada através de um maior número de amostras ou uma modificação no software para diminuir o intervalo entre duas freqüências consecutivas. Além disso, a imunidade a ruídos de um Analisador de Espectro Lock-in é muito superior a das técnicas convencionais de filtragem de sinal. A melhor resolução espectral em relação à FFT deve-se ao fato da técnica de detecção sensível à fase permitir a recuperação de um sinal em uma freqüência arbitrária (Meade, 1983), não havendo necessidade desta freqüência ser múltipla de uma constante de resolução espectral.

Para realizar a implementação digital do Analisador de Espectro Lock-in, foi utilizado um DSK (Developers Starter Kit) da Texas Instruments. O DSK é uma placa contendo um DSP, os componentes básicos para a sua utilização e um codec que atua como conversor analógico / digital e digital / analógico de 16 bits com taxa de amostragem máxima de $11025 \mathrm{~Hz}$, podendo-se alterar esta taxa via software. Esta placa é conectada a um PC pela porta paralela, permitindo sua programação em linguagem C ou Assembly através de um ambiente integrado de desenvolvimento (IDE).

O software desenvolvido para o DSP gera duas seqüências periódicas em quadratura (Meade, 1983). O componente em fase (cosseno) é usado para excitar ou modular o sinal de entrada de um sistema externo (experimento). Este 
componente é denominado sinal de referência. A freqüência da resposta do experimento é igual à da excitação do sinal de referência, desconsiderando componentes espúrios tais como o ruído branco.

\section{ESTIMAÇÃO INDIRETA DE VELOCIDADE DE UM MOTOR DE INDUÇÃO TRIFÁSICO}

O uso de métodos invasivos de medição da velocidade do eixo de um motor de indução trifásico tais como tacômetro com acoplamento mecânico ou com sensor ótico ou magnético podem ser inconvenientes ou pouco práticos. Em algumas instalações, por exemplo, não se tem acesso às extremidades do eixo do motor, dificultando a utilização de tacômetro com acoplamento mecânico. O uso de tacômetros baseados em sensores óticos ou mecânicos pode implicar na fixação de elementos ao eixo do motor (fita ótica ou disco dentado), o que nem sempre é possível e pode ter limitações de exatidão.

Neste contexto, métodos indiretos para estimar a velocidade do eixo apresentam-se como uma alternativa que não exige alterações na estrutura do motor nem interação mecânica com o mesmo (Hurst, 1996). Abordagens que utilizam o espectro de freqüências das correntes de um motor de indução trifásico enquadram-se na categoria de métodos não invasivos. A partir de freqüências relacionadas com a freqüência fundamental de alimentação (ex. : $60 \mathrm{~Hz}$ ) podese determinar a velocidade do eixo de um motor de indução trifásico em regime estacionário.

Para obter a velocidade do eixo de um motor de indução trifásico, inicialmente é realizada a aquisição de uma de suas correntes de fase através de um conversor analógicodigital. Posteriormente, o software desenvolvido para o DSP detecta uma freqüência $\left(f_{H}\right)$ cujo componente possui maior amplitude dentro de uma faixa de freqüências que depende das características do MIT e de parâmetros adotados (Hurst, 1996). Para um motor de 4 pólos e 44 ranhuras, em condições normais de operação, existe um componente de amplitude máxima entre o $21^{\circ}$ e o $23^{\circ}$ harmônicos da freqüência fundamental $\left(f_{F}\right)$ de alimentação da rede.

O número do harmônico máximo da faixa (Santana, 2000) pode ser calculado como:

$$
h_{\text {máximo }}=\frac{k}{(p / 2)}+1
$$

Onde $k$ é o número de ranhuras do motor de indução trifásico e $p$ é o número de pólos do motor de indução trifásico.

A partir destes dados, a razão entre a freqüência que possui a maior amplitude e a freqüência fundamental pode ser calculada como:

$$
h=\frac{f_{H}}{f_{F}}
$$

A expressão para calcular o escorregamento $s$ do eixo do motor de indução trifásico é (Hurst, 1996) :

$$
s=1-\frac{(p / 2) \cdot(h-1)}{k}
$$

Por exemplo, uma vez calculada a razão $h$ a partir de (6), o escorregamento $s$ do eixo do motor de indução trifásico, para um motor de 4 pólos e 44 ranhuras pode ser estimado através da expressão :

$$
s=1-\frac{(h-1)}{22}
$$

A partir do escorregamento s obtido em (8), estima-se a velocidade do eixo do motor de indução trifásico :

$$
n=n_{S} \cdot(1-s) \quad[\mathrm{rpm}]
$$

Onde $n_{S}$ é a velocidade síncrona do motor de indução trifásico que é igual a $1800 \mathrm{rpm}$ para 4 pólos (Munõz, 1987)

\section{DESCRIÇÃO DO ALGORITMO}

Para implementar o Analisador de Espectro Lock-in Digital, foi necessário desenvolver um software em linguagem C (Schildt, 1997) para ser executado pelo DSP e realizar a operação de recuperação sensível à fase de sinal.

O algoritmo desenvolvido para implementar o Analisador de Espectro Lock-in Digital consiste em realizar a recuperação lock-in em cada uma das freqüências em um intervalo, variando em passos discretos. O fluxograma simplificado do algoritmo é mostrado na Figura 3. 


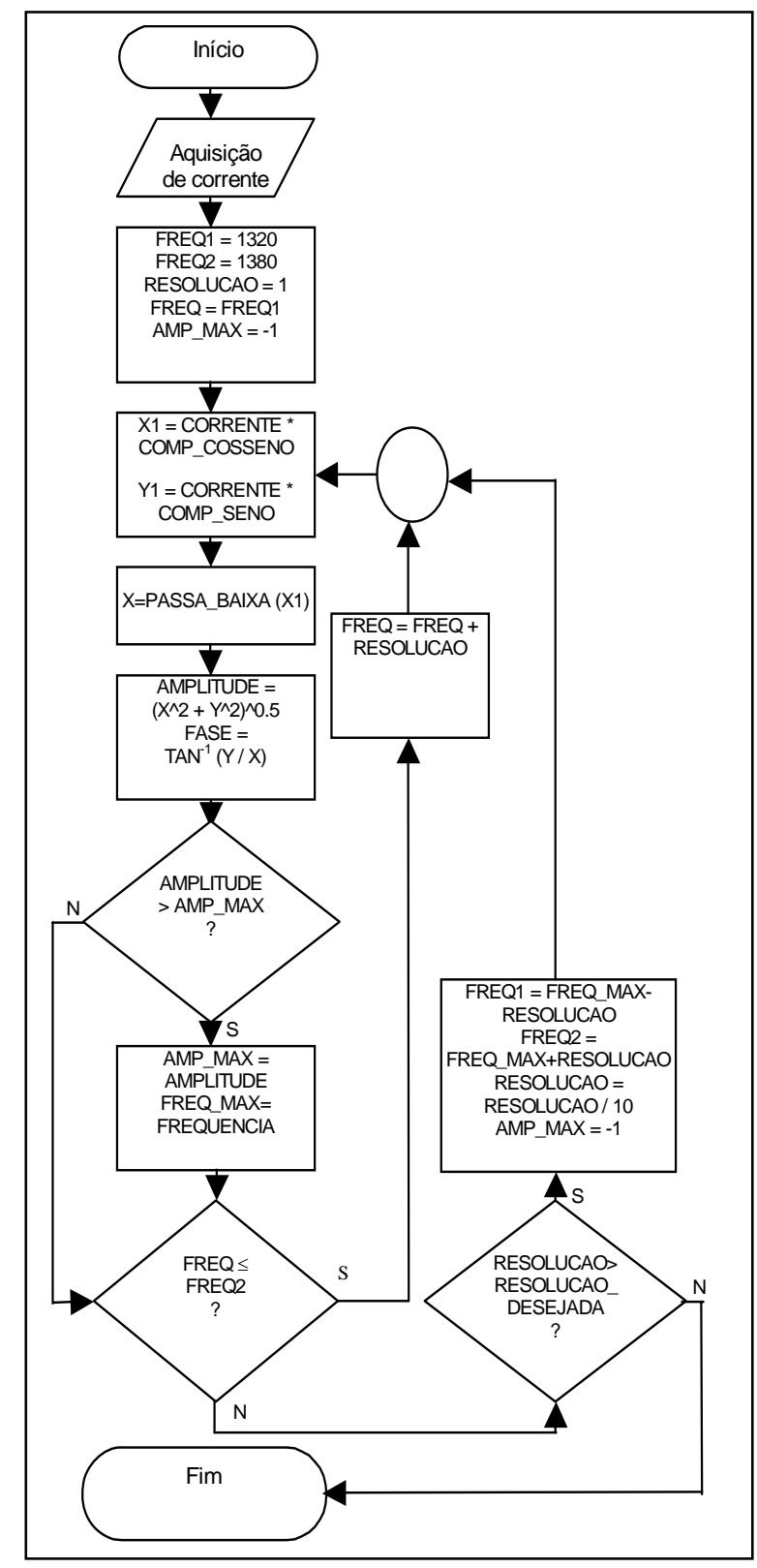

Figura 3 : Fluxograma do algoritmo do Analisador de Espectro Lock-in Digital.

\section{RESULTADOS EXPERIMENTAIS}

Um motor de indução trifásico de quatro pólos, potência nominal de $2 \mathrm{cv}$, corrente nominal de 6,5 A, velocidade nominal de $1725 \mathrm{rpm}$ e velocidade síncrona de $1800 \mathrm{rpm}$ foi utilizado nos ensaios experimentais. Estes ensaios foram realizados exclusivamente para obter dados que permitissem testar a técnica desenvolvida. Por meio de um sensor de efeito Hall usado como amperímetro, foi realizada a aquisição de uma das correntes de fase. Esta corrente é digitalizada por meio do codec do DSK para posterior processamento pelo DSP. Um gerador DC simula uma carga no motor de indução trifásico que pode ser graduada através de um potenciômetro. Esta carga é definida como a razão entre a corrente medida e a corrente nominal, sendo esta razão multiplicada por cem para obter o valor percentual da carga. Para as diferentes cargas foram realizadas medições de velocidade com um tacômetro ótico e estimações de velocidade utilizando a FFT e um Analisador de Espectro Lock-in Digital. A taxa de amostragem configurada para o conversor A/D de 16 bits é de $8 \mathrm{kHz}$ e foi realizada a aquisição de 2048 amostras. O conversor D/A de 16 bits também foi configurado para uma taxa de amostragem de $8 \mathrm{kHz}$. O passo de varredura em freqüência do Analisador de Espectro Lock-in é de 0,1 Hz. O filtro passa-baixas utilizado é do tipo IIR (Infinite Impulse Response) com dois coeficientes, um destes $(0,95)$ é multiplicado pela amostra atual e outro $(0,05)$ é multiplicado pela saída anterior do filtro, sendo a soma destas duas multiplicações a saída atual do filtro. Poderia também adotar-se uma média dos valores de $\mathrm{V}$ e $\phi$ ao longo do tempo, uma vez que a média funciona como um filtro passa-baixas. O tempo de acomodação dos filtros é dependente do sinal digitalizado de uma das correntes de fase do motor e pode-se definir como limite superior de tempo para convergência o tempo de aquisição de 2048 amostras (0,25 segundos). O tempo aguardado para estabilização do motor para cada velocidade foi de um minuto. A velocidade obtida do eixo do motor de indução trifásico pelos diferentes métodos pode ser vista na Tabela 1.

Tabela 1 - Velocidade obtida do eixo do Motor de indução trifásico através de FFT, tacômetro ótico e Analisador de Espectro Lock-in

\begin{tabular}{|c|c|c|c|}
\hline Carga & FFT & $\begin{array}{c}\text { Tacômetro } \\
\text { ótico } \\
(\%)\end{array}$ & $\begin{array}{c}\text { Analisador de } \\
\text { Espectro } \\
\text { Lock-in } \\
\text { (rpm) }\end{array}$ \\
\hline 53,07 & 1793,18 & 1793 & 1793,18 \\
\hline 54,31 & 1789,77 & 1789 & 1789,09 \\
\hline 56,62 & 1786,36 & 1788 & 1787,73 \\
\hline 62,46 & 1779,54 & 1780 & 1780,91 \\
\hline 68,46 & 1772,77 & 1772 & 1772,73 \\
\hline 74,92 & 1762,54 & 1763 & 1763,18 \\
\hline 81,23 & 1752,27 & 1752 & 1752,27 \\
\hline 87,54 & 1742,05 & 1742 & 1742,73 \\
\hline 93,08 & 1731,82 & 1733 & 1733,18 \\
\hline 98,15 & 1725 & 1725 & 1725 \\
\hline 99,69 & 1718,18 & 1718 & 1718,18 \\
\hline 184,62 & $*$ & 1376 & 1375,91 \\
\hline 194,12 & $*$ & 1347 & 1347,23 \\
\hline
\end{tabular}

* Não foi possível determinar a velocidade pela FFT 
Um tacômetro ótico digital com precisão de $\pm(0,05 \%+1$ dígito) e resolução de $1 \mathrm{rpm}$ acima de $1000 \mathrm{rpm}$ foi adotado como padrão de referência. A partir dos dados da Tabela 1, o erro quadrático médio usando a FFT é de 0,53 enquanto que o erro quadrático médio do Analisador de Espectro Lock-in é de 0,17 , ou seja, três vezes menor. Observou-se também que a FFT não permite estimar a velocidade para carregamentos acima de 184,62 \%, devido ao surgimento de componentes relacionados ao funcionamento do motor que dificultam a detecção correta de $f_{H}$. A variância das medidas após a estabilização do motor foi considerada desprezível para efeitos práticos, o que implica em repetibilidade consistente do método. A resolução espectral do Analisador de Espectro Lock-in pode ainda ser aumentada, com conseqüente aumento do tempo de processamento.

\section{CONCLUSÃO}

Neste artigo, foi usado um Analisador de Espectro Lock-in Digital para obter o espectro de freqüências de uma das correntes de fase de um motor de indução trifásico, abordagem esta que se mostrou mais exata e imune a ruídos do que as convencionais tais como a FFT e a PSD. A partir deste espectro, estimou-se de modo não-invasivo a velocidade do eixo de um motor de indução trifásico. O tempo de processamento para a estimação da velocidade do eixo do motor foi de três segundos, o que torna a técnica mais adequada para aplicações onde a exatidão é mais importante do que o tempo de estimação da velocidade. Pode-se reduzir o tempo de processamento por meio da aplicação da FFT para obter uma estimativa inicial da velocidade, posteriormente refinando esta estimativa com o Analisador de Espectro Lock-in Digital.

\section{REFERÊNCIAS BIBLIOGRÁFICAS}

Blasco-Gimenez, R., Asher, G. M., Sumner, M., Bradley, K. J. (1996). Performance of FFT-rotor slot harmonic speed detector for sensorless induction motor drives, IEE Proceedings, Vol. 143, Issue 3

Hurst, K. D. H., Habetler, T. G. (1996). Sensorless Speed Measurement Using Current Harmonic Spectral Estimation in Induction Machine Drives", IEEE Transactions on Power Electronics, Vol. 11, Number 1

Meade, M. (1983). Lock-in amplifiers : principles and applications, Short Run Press Ltd
Muñoz, N. T. (1987). Cálculo de enrolamentos de máquinas elétricas e sistemas de alarme", Biblioteca Técnica Freitas Bastos.

Oppenheim, A. V., Willsky A. S. (1997). Signal and Systems, Prentice-Hall.

Press, W. H. et al, (1992). Numerical Recipes, Cambridge University Press.

Santana, A. M. S., Lima, A.C.C., Aguiar, A. L. (2000). Estimação de Velocidade de Motores de Indução Trifásicos, Universidade Federal da Bahia, Eletricidade Moderna, março / 2000

Schildt, H. (1997). C Completo e total, Mcgraw-Hill

Stark, H., Woods, J. H. (1998). Probability, Random Processes and Estimation Theory for Engineers, Prentice Hall.

Tompkins, W. J. (1993). Biomedical Digital Signal Processing, Prentice Hall 